Marquette University

e-Publications@Marquette

$8-12-1997$

\title{
Spectroscopically Distinct Cobalt(II) Sites in Heterodimetallic Forms of the Aminopeptidase from Aeromonas proteolytica: Characterization of Substrate Binding
}

Brian Bennett

Marquette University, brian.bennett@marquette.edu

Richard C. Holz

Marquette University, richard.holz@marquette.edu

Accepted version. Biochemistry, Vol. 36, No. 32 (August 12, 1997): 9837-9846. DOI. (C) 1997 American Chemical Society. Used with permission.

Brian Bennett and Richard C. Holz were affiliated with Utah State University at the time of publication. 


\section{Marquette University}

\section{e-Publications@Marquette}

\section{Physics Faculty Research and Publications/College of Arts and Sciences}

This paper is NOT THE PUBLISHED VERSION; but the author's final, peer-reviewed manuscript. The published version may be accessed by following the link in th citation below.

Biochemistry, Vol. 36, No. 32 (1997): 9837-9846. DOI. This article is (C) American Chemical Society and permission has been granted for this version to appear in e-Publications@Marquette. American Chemical Society does not grant permission for this article to be further copied/distributed or hosted elsewhere without the express permission from American Chemical Society.

\section{Spectroscopically Distinct Cobalt(II) Sites in Heterodimetallic Forms of the}

\section{Aminopeptidase from Aeromonas proteolytica: Characterization of Substrate Binding ${ }^{\dagger}$}

Brian Bennett

Department of Chemistry and Biochemistry, Utah State University, Logan, Utah

Richard C. Holz

Department of Chemistry and Biochemistry, Utah State University, Logan, Utah

\footnotetext{
Abstract

The Co(II)Zn(II)- and Zn(II)Co(II)-substituted derivatives of the aminopeptidase from Aeromonas proteolytica (AAP) were probed by EPR spectroscopy. EPR spectra of the high-spin $S=3 / 2$ Co(II) ions in [CoZn(AAP)] and [ZnCo(AAP)] indicated that each metal binding site provides a spectroscopically distinct signature. For [CoZn(AAP)], subtraction of EPR spectra recorded at $\mathrm{pH} 7.5$ and 10 revealed that two species were present and that the relative contributions to each of the experimental spectra were $\mathrm{pH}$-dependent. The first EPR species, predominant at lower $\mathrm{pH}$ values, was simulated as a relatively featureless axial signal with $g_{\text {eff }}$ values of $2.20,3.92$, and 5.23 which correspond to an $M_{s}=| \pm 1 / 2\rangle$ ground state transition with a $g_{\text {real }}$ of 2.29 and an $E / D$ of 0.1 . The second species, predominant at high $\mathrm{pH}$, was simulated with $g_{\text {eff }}$ values of $1.80,2.75$, and 6.88 and exhibited a characteristic eight-line ${ }^{59} \mathrm{Co}$ hyperfine pattern with an $A_{2}\left({ }^{59} \mathrm{Co}\right)$ of $7.0 \mathrm{mT}$. These
} 
parameters correspond to an $M_{\mathrm{s}}=| \pm 1 / 2\rangle$ ground state transition with a $g_{\text {real }}$ of 2.54; however, the signal exhibited marked rhombicity $(E / D=0.32)$ indicative of an asymmetric tetrahedral or five-coordinate Co(II) ion. Summation of these two species provided an excellent simulation of the observed [CoZn(AAP)] EPR spectrum. The EPR spectrum of [ZnCo(AAP)] also contained two species, at least one of which also exhibited ${ }^{59} \mathrm{Co}$ hyperfine features. However, this signal exhibited little $\mathrm{pH}$ dependence, and individual species could not be isolated. The addition of the competitive inhibitor 1-butaneboronic acid (BuBA) to [CoZn(AAP)] resulted in a distinct change in the EPR spectrum; however, addition of BuBA to [ZnCo(AAP)] left the EPR spectrum completely unperturbed. These data indicate that BuBA binds only to the first metal binding site in AAP and does not interact with the second site. On the basis of the X-ray crystallographic data for the transition state analog-inhibited complexes of AAP and the aminopeptidase from bovine lens, BuBA was reclassified as a substrate analog inhibitor rather than a transition state analog inhibitor as previously suggested [Baker, J. O., \& Prescott, J. M. (1983) Biochemistry 22, 5322-5331]. From difference spectroscopy and from the simulation of the [CoZn(AAP)] EPR spectrum, a third signal appearing upon BuBA binding was isolated. This signal was simulated with $g_{\text {eff }}$ values of $2.08,3.15$, and 6.15 which correspond to an $M_{s}=| \pm 1 / 2\rangle$ ground state transition with a $g_{\text {real }}$ of 2.41 and an $E / D$ of 0.22 . This simulation also invoked an eight-line unresolved ${ }^{59} \mathrm{Co}$ hyperfine pattern with an $A_{z}\left({ }^{59} \mathrm{Co}\right)$ value of $4.0 \mathrm{mT}$. Summation of the these three species provided an excellent simulation of the observed [CoZn(AAP)] + BuBA EPR spectrum at both $\mathrm{pH}$ values. This work establishes that substrate binds only to the first metal binding site in AAP and thus substantiates the first step in catalysis in the recently proposed mechanism of action for AAP [Bennett, B., \& Holz, R. C. (1997) J. Am. Chem. Soc. 119, 1923-1933; Chen, G., et al. (1997) Biochemistry 36, 4278-4286]. Di- and trinuclear metallohydrolases play important roles in hydrolyzing some of the most important molecules in life such as DNA, phospholipids, and polypeptides (Dismukes, 1996; Lipscomb \& Sträter, 1996; Sträter et al., 1996; Wilcox, 1996). They are therefore key players in carcinogenesis, tissue repair, and protein degradation processes. In addition, dinuclear metallohydrolases are involved in the degradation of agricultural neurotoxins, antibiotics, and several phosphorus(V) materials used in chemical weaponry (Menger et al., 1987; Chin, 1991; Lai et al., 1994). Recent structural studies have shown that many dinuclear metallohydrolases are also members of a class of enzymes that contain carboxylate rich coordination environments (Lipscomb \& Sträter, 1996; Sträter et al., 1996; Wilcox, 1996). Some of the enzymes in this group include phospholipase C (Hough et al., 1989), alkaline phosphatase (Kim \& Wyckoff, 1991), inositol monophosphatase (Bone et al., 1994), DNA polymerase I (Beese \& Steitz, 1991), the ribonuclease H domain of HIV-1 reverse transcriptase (Davies et al., 1991), P1 nuclease (Lahm et al., 1990), urease (Jabri et al., 1995), the purple acid phosphatases (Holz et al., 1992; True et al., 1993; Sträter et al., 1995), calcineurin (Griffith et al., 1995), and the aminopeptidases (Burley et al., 1990, 1992; Roderick \& Matthews, 1993; Chevrier et al., 1994). Despite their ubiquity and the wealth of structural information available, little is known about how these structural motifs relate to function.

Multinuclear metallohydrolases utilize a wide variety of metal ions in catalysis that include all of the first row transition metal ions from $\mathrm{Mn}(\mathrm{II})$ to $\mathrm{Zn}(\mathrm{II})$, except $\mathrm{Cu}(\mathrm{II})$. The structural aspects of these sites (e.g. coordination geometry and ligand type) appear to influence the nature of the hydrolytic reaction and represent at least one determinant of the reaction mechanism. Only a handful of dinuclear metallohydrolases contain or can utilize heterodimetallic active sites. Included among the crystallographically characterized enzymes are the Klenow fragment of DNA polymerase I (Escherichia coli) (Beese \& Steitz, 1991), alkaline phosphatase (E. coli) (Kim \& Wyckoff, 1991), DNA polymerase (Thermus aquaticus) (Kim et al., 1995), leucine aminopeptidase (bovine lens) (Kim \& Lipscomb, 1993), purple acid phosphatase (kidney bean) (Sträter et al., 1995), and calcineurin (Griffith et al., 1995). Other notable noncrystallographically characterized heterodimetallic examples include uteroferrin (Lauffer et al., 1983; Holz et al., 1992; True et al., 1993) and the

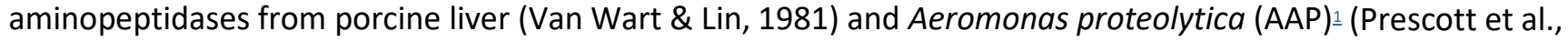
1985). These enzymes use different metal ion Lewis acidities in discrete heterodimetallic sites to (i) bind and position substrate, (ii) bind and activate a water molecule to yield an active site hydroxide nucleophile, and/or (iii) stabilize the transition state of the hydrolytic reaction. 
Heterodimetallic centers in metallohydrolases provide systems in which the function of each metal ion in catalysis can be uniquely probed through independent labeling of each metal binding site with a spectroscopically active and silent metal ion, respectively. However, heterodimetallic metal-substituted enzymes in which an active enzyme is obtained are extremely rare. Notable examples are the purple acid phosphatases from porcine uterus and kidney bean which contain Fe(III)/Zn(II) cores (Lauffer et al., 1983; Holz et al., 1992; True et al., 1993; Sträter et al., 1995). However, only the Fe(III) site can be probed spectroscopically since removal or reduction of the Fe(III) center results in complete loss of enzymatic activity. The aminopeptidase from A. proteolytica (AAP) possesses ideal biological properties for spectroscopically probing hydrolase activity catalyzed by dinuclear metal centers (Prescott \& Wilkes, 1976). AAP is a small, monomeric enzyme (29 $500 \mathrm{Da})$, containing $2 \mathrm{~mol}$ of zinc per mole of polypeptide. It is thermostable for several hours at $70{ }^{\circ} \mathrm{C}$ (Prescott \& Wilkes, 1976) and has been crystallographically characterized (Chevrier et al., 1994). Substitution of the 2 mol atoms of zinc in AAP with $\mathrm{Co}(\mathrm{II}), \mathrm{Cu}(\mathrm{II})$, or $\mathrm{Ni}(\mathrm{II})$ provides different magnitudes of activity that are dependent on the sequence and order of addition (Prescott et al., 1983, 1985; Bayliss \& Prescott, 1986). For example, the Cu(II)-, $\mathrm{Co}(\mathrm{II})$-, and $\mathrm{Ni}(\mathrm{II})$-substituted enzymes are hyperactive by $6.5,7.7$, and 25 times, respectively, toward certain substrates. Bennett and Holz (1997) recently demonstrated that metal binding to AAP occurs in a sequential fashion, highlighting the potential for the formation of heterodimetallic sites in AAP. The addition of 1 mol of $\mathrm{CU}(\mathrm{II}), \mathrm{Co}(\mathrm{II})$, or $\mathrm{Ni}$ (II) to apo-AAP followed by the addition of $\mathrm{Zn}$ (II) provides a discrete heterodimetallic active site that is enzymatically active in each case. For $\mathrm{Ni}(\mathrm{II})$ and $\mathrm{Cu}(\mathrm{II})$, nearly 90 - and 100 -fold increases in activity ae observed, respectively (Prescott et al., 1983, 1985). Therefore, AAP provides a unique system with which to probe the role of each metal ion in catalysis by a wide variety of transition metal ions in discrete heterodimetallic sites.

In an effort to understand the role of each metal ion in catalysis and thus gain further insight into the structure and function of dinuclear hydrolases, we have examined the Co(II) Zn(II)- and Zn(II)Co(II)-substituted forms of the aminopeptidase from A. proteolytica. EPR and electronic absorption spectra of [CoZn(AAP)] and [ZnCo(AAP)] indicate that each metal binding site provides a spectroscopically distinct signature. Therefore, EPR spectroscopy can be used to selectively probe each metal binding site. EPR spectra were simulated using a new systematic protocol for the analysis of high-spin $S=3 / 2 \mathrm{Co}$ (II) EPR spectra (Bennett \& Holz, 1997). Analysis of these distinct EPR spectra in the presence and absence of the competitive inhibitor 1-butaneboronic acid (BuBA) establishes that BuBA is in fact a substrate analog inhibitor. We have also investigated the effect of $\mathrm{pH}$ on the observed $S=3 / 2$ signals both in the absence and in the presence of BuBA in order to probe the potentially important ionizable groups associated with the dimetal cluster. These data establish that substrate binds only to the first metal binding site in AAP and thus substantiate the first step in catalysis in the recently proposed mechanism of action for AAP (Bennett \& Holz, 1997; Chen et al., 1997).

\section{Materials and Methods}

Enzyme Purification. All chemicals used in this study were purchased commercially and were of the highest quality available. The aminopeptidase from $A$. proteolytica was purified from a stock culture kindly provided by C. Schalk. Cultures were grown according to the previously published procedure (Prescott \& Wilkes, 1976) with minor modifications to the growth media (Chen et al., 1997). AAP was routinely purified, quantitated, and assayed as described in detail elsewhere (Bennett \& Holz, 1997).

Metal-Substituted Sample Preparation. [CoZn(AAP)] and [ZnCo(AAP)] were prepared by a method similar to that reported by Prescott et al. (1985). Briefly, AAP was dialyzed for $72 \mathrm{~h}$ at $4{ }^{\circ} \mathrm{C}$ against $10 \mathrm{mM} 1,10-$ phenanthroline monohydrochloride in $50 \mathrm{mM}$ Hepes buffer at $\mathrm{pH} 7.5$ and then exhaustively dialyzed against Chelex-treated Hepes buffer. Metal insertion was effected by direct addition, with efficient mixing of 1 equiv of $\mathrm{MCl}_{2}$ (where $\mathrm{M}=\mathrm{Co}$ or $\mathrm{Zn}$; $\geq 99.999 \% \mathrm{CoCl}_{2}$, Strem Chemicals, Newburyport, $\mathrm{MA} ; \mathbf{9 9 . 9 9 9 \% ~} \mathrm{ZnCl}_{2}$, Aldrich) followed by a $30 \mathrm{~min}$ incubation period at $20-25{ }^{\circ} \mathrm{C}$. The second metal was then inserted in the same manner, and the samples were frozen in liquid nitrogen prior to spectroscopic studies. Samples for study at pH 10 were 
generated as above in Hepes buffer at pH 7.5 and then anaerobically dialyzed against $50 \mathrm{mM}$ Caps buffer, pH 10 . [ZnZn(AAP)], [CoCo(AAP)], [CoZn(AAP)], and [ZnCo(AAP)] were assayed for activity with L-leucine- $p$-nitroanilide and L-alanine-p-nitroanilide $\left(\Delta \varepsilon_{405}\right.$ value of $p$-nitroaniline of $\left.10800 \mathrm{M}^{-1} \mathrm{~cm}^{-1}\right)$, and the activities obtained were entirely in agreement with those reported earlier (Prescott et al., 1983, 1985). For example, the specific activity of the dizinc(II) enzyme compared to that of the dicobalt(II) enzyme toward the substrate L-leucine- $p$ nitroanilide was 120 and 24 units, respectively. The dicobalt(II) enzyme is hyperactive toward the substrate Lalanine- $p$-nitroanilide compared to the dizinc(II) enzyme with specific activities of 0.9 and 0.2 unit, respectively. Also in agreement with earlier work (Prescott et al., 1985), the enzyme was found to attain $~ 80 \%$ of the native activity upon the addition of only 1 equiv of Co(II) or $\mathrm{Zn}(\mathrm{II})$.

The addition of butaneboronic acid (BuBA) was effected by incubating approximately 10 equiv with AAP samples for short times ( $\sim 10 \mathrm{~s})$. Briefly, $\sim 20 \mu \mathrm{L}$ of $100 \mathrm{mM}$ BuBA was introduced onto the inside wall of an EPR tube and the enzyme sample introduced above this as a plug $\sim 2 \mathrm{~cm}$ length. Rapid mixing was effected with one sharp motion, and the samples were frozen rapidly in a dry ice/methanol bath. Using control solutions which develop color upon mixing, this technique has previously been shown to result in efficient mixing (Bennett et al., 1994a,b).

Spectroscopic Measurements. Electronic absorption spectra and activity assays were carried out on a Shimadzu UV-3101PC spectrophotometer equipped with a constant-temperature holder and a Haake (type 423) constant-temperature circulating bath. Subtraction of the absorption spectrum of apo-AAP from that of metalsubstituted enzyme samples was performed using Shimadzu UV-3101 software. Low-temperature EPR spectroscopy was performed using a Bruker ESP-300E spectrometer equipped with an ER 4116 DM dual-mode Xband cavity and an Oxford Instruments ESR-900 helium flow cryostat as described previously (Bennett \& Holz, 1997). Background spectra recorded on a buffer sample were aligned with and subtracted from experimental spectra as in earlier work (Bennett et al., 1994a,b, 1995). Signals at low temperatures due to oxygen were occasionally observed in both EPR modes. These signals were abolished upon raising the temperature in the helium cryostat to $125 \mathrm{~K}$ for $5 \mathrm{~min}$ and recooling. All spectra were recorded at a modulation frequency of 100 $\mathrm{kHz}$, a modulation amplitude of $1.26 \mathrm{mT}(12.6 \mathrm{G})$, and a sweep rate of $10 \mathrm{mT} \mathrm{s}^{-1}$. All EPR spectra were recorded at microwave frequencies of approximately $9.65 \mathrm{GHz}$; precise microwave frequencies were recorded for individual spectra to ensure precise galignment. Other EPR running parameters are specified in the figure legends for individual samples. Enzyme concentrations for EPR studies were typically 1-2 mM. All buffers contained $\sim 20 \%$ 2-propanol to prevent aggregation at high protein concentrations. Purified enzyme stored for up to 2 weeks at 4 ${ }^{\circ} \mathrm{C}$ in Hepes buffer (pH 7.5) containing 20\% 2-propanol (by volume) showed no measurable decrease in activity. EPR spectra recorded on analogous [CoCo(AAP)] samples except for the presence and absence of $20 \% 2$ propanol were indistinguishable. Computer techniques for resolution enhancement were employed to aid in the simulation of ${ }^{59} \mathrm{Co}$ hyperfine structure and have been described in detail elsewhere (Sears et al., 1995).

Computer Simulations of High-Spin Co(II) $S=3 / 2$ EPR Spectra. The protocol for simulation of high-spin Co(II) EPR spectra was described in detail elsewhere (Bennett \& Holz, 1997). Briefly, $g_{\text {eff }(x, y, z)}$ values were obtained by simulation of $S=3 / 2$ high-spin Co(II) species by treating the system as an effective $S=1 / 2$ system (Figure 1 ) and using the program EPRSim XOP for Igor Pro by J. Boswell (Oregon Graduate Institute) (Belford, 1979; Nilges, 1979; Maurice, 1980). $g_{\text {real }}$ values and rhombicities $(E / D)$ were extracted from the $g_{\text {eff }}$ values using eqs $1-3$ (Pilbrow, 1978) and the program Rhombogram, kindly supplied by W. R. Hagen:

$$
\begin{gathered}
g_{\text {eff(x) }}=g_{\text {real }(x)}\left[1 \pm[1+3(E / D)] / \sqrt{ }\left[1+3(E / D)^{2}\right]\right](1) \\
g_{e f f_{(y)}}=g_{\text {real }(y)}\left[1 \pm[1-3(E / D)] / \sqrt{ }\left[1+3(E / D)^{2}\right]\right](2)
\end{gathered}
$$




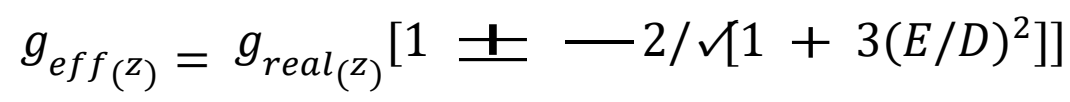

The relationships between $g_{\text {eff }}, g_{\text {real, }}$ and $E / D$ are illustrated graphically in Figure 2. In all simulations, an isotropic $g_{\text {real }}$ value was assumed.
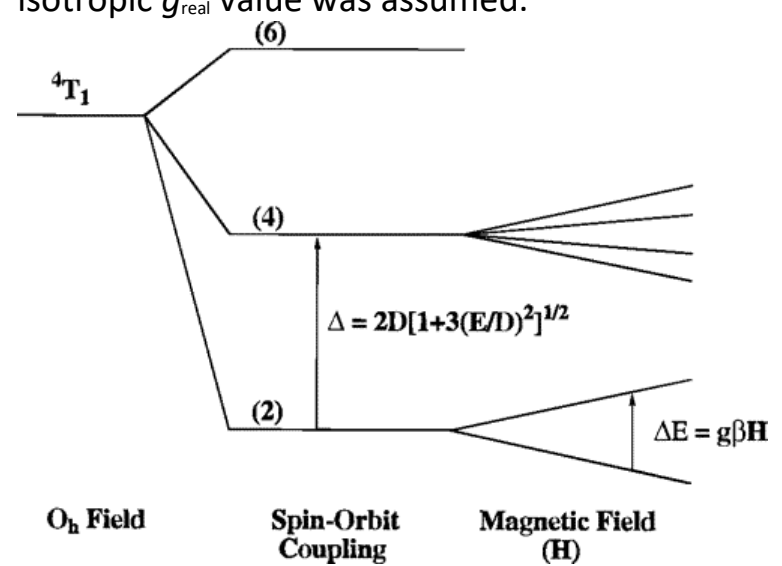

Figure 1 Energy level diagram for an effective $S=1 / 2 \mathrm{Co}(I I)$ ion in an octahedral field.
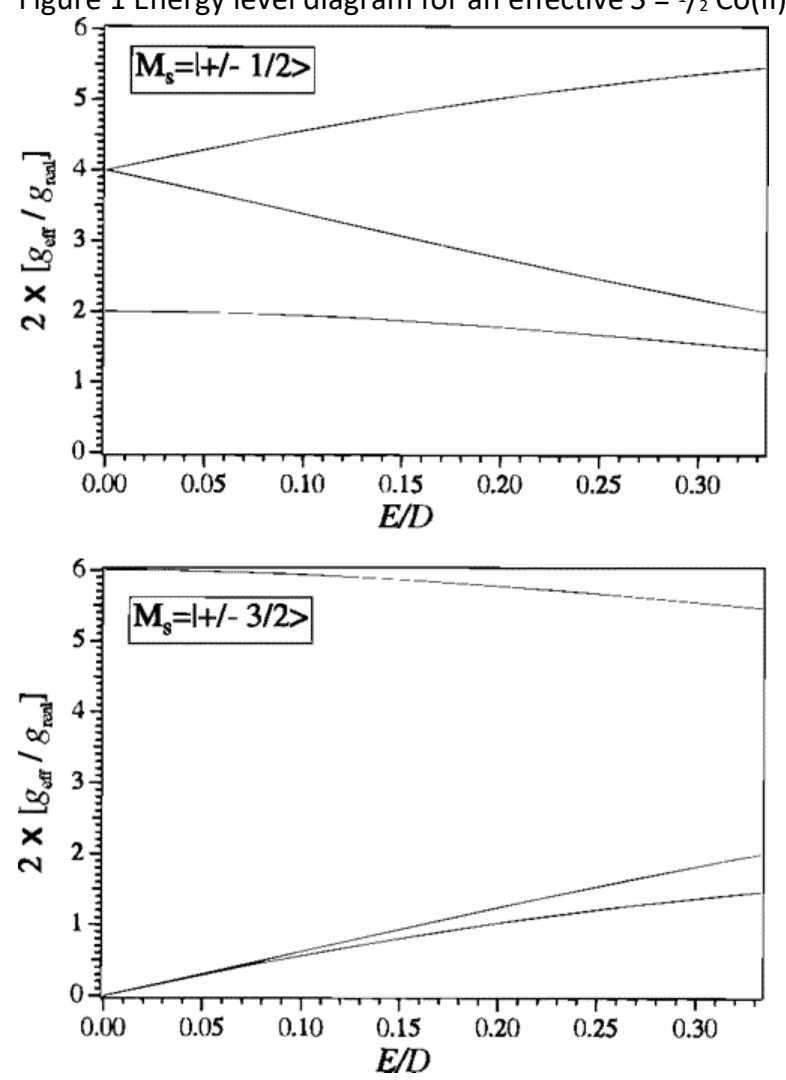

Figure 2 Generalized rhombograms for the $M_{s}=| \pm 1 / 2\rangle$ and $M_{s}=| \pm 3 / 2\rangle$ manifolds of an $S=3 / 2$ spin system for which $g_{\text {real }}$ is not necessarily 2.0. The abscissa is plotted in units of $2\left(g_{\text {eff }} / g_{\text {real }}\right)$ so that the values of $g_{\text {eff }}$ can be directly determined from the rhombogram when $g_{\text {real }}=2.0$.

This method of analysis yields two sets of $g_{\text {eff }}$ values: those obtained by simulation of the line shape and those allowed by theory. The latter values are given in parentheses after the former. Comparison between these two sets of $g_{\text {eff }}$ values gives a quantitative indication of the validity of the simulation. The small observed discrepancies are probably due to anisotropy of $g_{\text {real }}$. Where spectra were due to more than one EPR active species, individual species were first isolated using standard difference spectroscopy techniques (Bray, 1988; Bennett et al., 1994a,b; Bennett \& Holz, 1997). Experimental spectra were simulated by the summation of the 
appropriate amounts of individual contributory species. Information on the zero-field splitting parameter, $\Delta$, could not be reliably obtained for reasons described in detail in earlier work (Bennett \& Holz, 1997). For spectra that were composed of more than one species, the relative contribution of each species was estimated by summing various proportions of the individual species simulations together and doubly integrating each of these simulations.

\section{Results}

Electronic Absorption Studies. Electronic absorption spectra were recorded for [CoZn(AAP)] and [ZnCo(AAP)] (Figure 3A), and the component due to apo-AAP was subtracted. The addition of 1 equiv of $\mathrm{Co}$ (II) followed by the addition of 1 equiv of $\mathrm{Zn}$ (II) provided a visible absorption spectrum with a broad band at $525 \mathrm{~nm}$ $\left(\varepsilon_{525} \sim 50 \mathrm{M}^{-1} \mathrm{~cm}^{-1}\right)$. This spectrum was very similar to that reported for [Co_(AAP)] (Prescott et al., 1985; Bennett \& Holz, 1997). However, addition of 1 equiv of $\mathrm{Zn}$ (II) followed by 1 equiv of Co(II) to apo-AAP provided an absorption spectrum with very little clear absorption around $525 \mathrm{~nm}$ due to $\mathrm{Co}(\mathrm{II})$. Most of the absorptivity of [ZnCo(AAP)] $\left(\varepsilon_{525} \sim 40 \mathrm{M}^{-1} \mathrm{~cm}^{-1}\right)$ appeared to be due to a broad underlying absorption band. A similar broad absorption band was observed for the Co(II)-substituted R2 subunit of ribonucleotide reductase (Elgren et al., 1994) and was also evident in several Co(II) model complexes (Bertini \& Luchinat, 1984). The residual absorption at $525 \mathrm{~nm}$ due to the second $\mathrm{Co}(\mathrm{II})$ ion is only $\sim 10 \mathrm{M}^{-1} \mathrm{~cm}^{-1}$. The molar absorptivities and absorption maxima of the electronic absorption spectra of [CoZn(AAP)] and [ZnCo(AAP)] are consistent with the first $\mathrm{Co}(\mathrm{II})$ ion residing in a tetrahedral or possibly five-coordinate environment, while the second $\mathrm{Co}(\mathrm{II})$ ion is octahedral. These spectra are essentially indistinguishable from those recorded by Prescott and co-workers which were supported by magnetic circular dichroism spectra (Prescott et al., 1985; Bennett \& Holz, 1997).
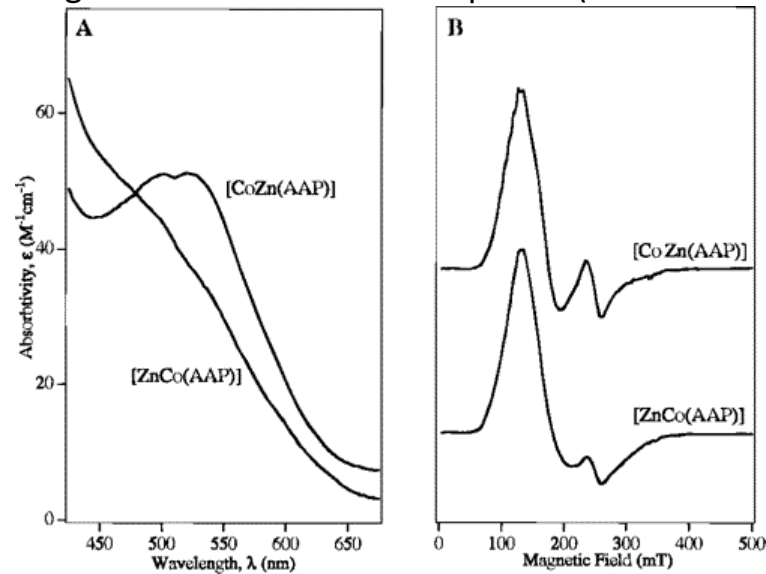

Figure 3 (A) Electronic absorption spectra of [CoZn(AAP)] and [ZnCo(AAP)]. The spectra have had the absorption component due to apo-AAP subtracted. (B) EPR spectra of [CoZn(AAP)] and [ZnCo(AAP)] at pH 7.5 in $50 \mathrm{mM}$ Hepes buffer and 20\% 2-propanol. Both spectra were recorded at $12 \mathrm{~K}$ with a $0.2 \mathrm{~mW}$ microwave power, a $1.26 \mathrm{mT}$ field modulation amplitude, a $100 \mathrm{kHz}$ modulation frequency, and a $10 \mathrm{mT} \mathrm{s}^{-1}$ field sweep rate.

EPR studies of [CoZn(AAP)] and [ZnCo(AAP)] at pH 7.5. The addition of 1 equiv of Co(II) to apo-AAP at $\mathrm{pH}$ 7.5 followed by the addition of 1 equiv of $\mathrm{Zn}(\mathrm{II})$ ([CoZn(AAP)]) provided a sample with an EPR spectrum consistent with a high-spin $S=3 / 2 \mathrm{Co}$ (II) center (Figure 3B). However, the eight-line hyperfine pattern due to the $I=7 / 2{ }^{59} \mathrm{Co}$ nucleus was not centered on the low-field absorption feature. This feature, along with the presence of the derivative-shaped feature at $243 \mathrm{mT}$, clearly indicated the presence of more than one signalgiving species, and not surprisingly, the signal could not be simulated as a single species. Similar complex signals have been observed for [Co_(AAP)] (Bennett \& Holz, 1997) as well as for several related enzymes; see for example the EPR spectra of Co(II)-substituted enolase (Rose et al., 1984), the Co(II)-substituted R2 subunit of ribonucleotide reductase (Elgren et al., 1994), and the $[\mathrm{Zn}(\mathrm{c}) \mathrm{Co}(\mathrm{n})]$ form of horse liver alcohol dehydrogenase (Werth et al., 1995). The EPR spectrum of AAP plus 1 equiv of $Z n(I I)$ followed by the addition of 1 equiv of $\mathrm{Co}$ (II) ([ZnCo(AAP)]) provided a sample with an EPR signal distinct from that observed for [CoZn(AAP)]. The minimum of the lower field feature, which crossed the baseline, was at a significantly higher field [214 mT for [ZnCo(AAP)] 
compared to $199 \mathrm{mT}$ for [CoZn(AAP)]] (Figure 3B). Furthermore, this feature was broader for [ZnCo(AAP)], and there was a very broad EPR absorption from 250 to $400 \mathrm{mT}$ that resulted in a marked asymmetry of the derivative feature at $\sim 250 \mathrm{mT}$. On the other hand, the analogous feature in the spectrum of [CoZn(AAP)] was well-formed and symmetric. A weak hyperfine pattern due to ${ }^{59} \mathrm{Co}$ was also observed for [ZnCo(AAP)] but was not centered on the low-field absorption feature, also indicating that more than one species was present. The differences between the EPR spectra of [CoZn(AAP)] and [ZnCo(AAP)] at pH 7.5 were subtle but corroborate the much more dramatic evidence from electronic absorption spectroscopy (Figure $3 \mathrm{~A}$ ) indicating that the two metal binding sites can be selectively labeled.

Differentiation of the two species observed in the EPR spectrum of [CoZn(AAP)] (Figure 4A) was achieved either by subtraction of EPR spectra recorded at various temperatures or microwave powers or by subtraction of spectra recorded on samples at different $\mathrm{pH}$ values. The first species (Figure 4C), accounting for $\sim 75 \%$ of the total signal intensity, was a relatively featureless axial signal with effective $g$ values for $g_{\text {eff }(x, y, z)}$ of 2.20 (2.22), 3.92 (3.87), and 5.23 (5.22) (Figure 4D) (the $g_{\text {eff }}$ values given are those that gave the best simulation, whereas those in parentheses are the closest allowed by theory; see Materials and Methods). These values correspond to an $M_{s}=| \pm 1 / 2\rangle$ ground state transition with a $g_{\text {real }}$ of 2.29 and an $E / D$ of 0.1 . This signal was similar to that observed for [CoCo(AAP)] as well as several other $\mathrm{Co}(\mathrm{II})$ systems except that the $g_{2}$ feature was unusually sharp compared to that for $[\mathrm{CoCo}(A A P)]$ or $\left[\mathrm{Co}\left(\mathrm{H}_{2} \mathrm{O}\right)_{6}\right]^{2+}$. Signals of this type are typical of $\mathrm{Co}(\mathrm{II})$ species with essentially axial symmetry (Bennett $\&$ Holz, 1997). The second species (Figure $4 \mathrm{E}$ ) had $g_{\text {eff }}$ values for $g_{\text {eff }(x, y, z, z)}$ of 1.80 (1.92), 2.75 (2.66), and 6.88 (6.88) and exhibited acharacteristic eight-line ${ }^{59} \mathrm{Co}$ hyperfine pattern with an $A_{z}\left({ }^{59} \mathrm{Co}\right)$ of $7.0 \mathrm{mT}$ (Figure $4 \mathrm{~F}$ ). These parameters correspond to an $M_{\mathrm{s}}=| \pm 1 / 2\rangle$ ground state transition with a $g_{\text {real }}$ of 2.54; however, the signal exhibited marked rhombicity $(E / D=0.32)$, consistent with, though not diagnostic of, an asymmetric tetrahedral or five-coordinate Co(II) ion. These data were also consistent with the previously reported electronic absorption spectra (Prescott et al., 1985). The possibility that the spectrum could be due to contributions from the $M_{s}=| \pm 1 / 2\rangle$ and $M_{s}=| \pm 3 / 2\rangle$ doublets of a single spin system was investigated; however, simulations consistent with theory could not be obtained; and difference analyses of experimental spectra clearly correlated the features at $g=2.75$ and $g=1.80$ with that at $g=6.88$. Theory dictates that the two lower $g$ values would be significantly less than unity and be barely observable if arising from an $M_{s}=| \pm 3 / 2\rangle$ doublet. Summation of these two species yields an excellent simulation of the observed [CoZn(AAP)] EPR spectrum (Figure 4B). Note that the small ripples in the simulation of the $g_{\text {real }}=2.54$ signal at $130-200 \mathrm{mT}$ (Figure $4 \mathrm{~F}$ ) were an artifact due to restrictions imposed by the simulation program on the number of intervals along $\theta$ and $\phi$ for which spectra were calculated. Because the individual species contributing to the spectrum of [ZnCo(AAP)] were not sufficiently sensitive to changes in the EPR running conditions or small changes in $\mathrm{pH}$, individual species contributing to the spectrum could not be isolated. Therefore, precise simulations could not be obtained, nor could the spectrum be simulated assuming a single species; however, a broad axial species appears to account for $\leq 85 \%$ of the total signal intensity.

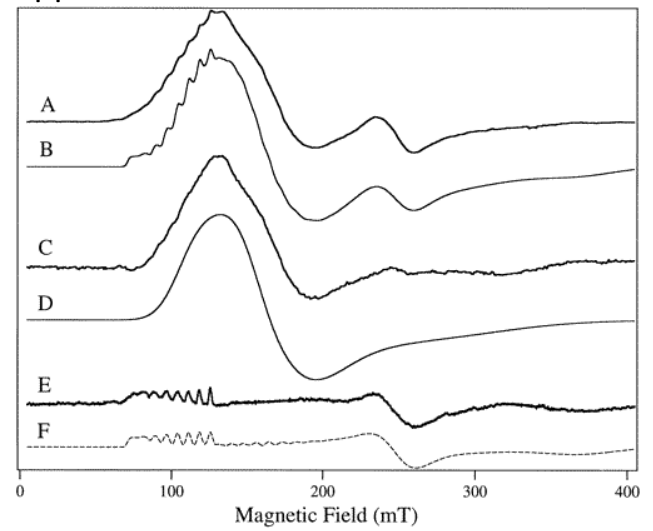

Figure 4 EPR spectra of [CoZn(AAP)] at pH 7.5 in $50 \mathrm{mM}$ Hepes buffer and 20\% 2-propanol. (A) EPR spectrum of [CoZn(AAP)] recorded at $12 \mathrm{~K}$ with a $0.2 \mathrm{~mW}$ microwave power, a $1.26 \mathrm{mT}$ field modulation amplitude, a $100 \mathrm{kHz}$ modulation frequency, and a $10 \mathrm{mT} \mathrm{s}^{-1}$ field sweep rate. (B) Simulation of spectrum A obtained by summing the individual simulations of spectra D and F. (C) 
EPR spectrum of an isotropic species obtained by subtraction of spectrum $E$ from A. (D) Simulation of spectrum $C$ with $g_{\text {eff }}$ values of $5.23,3.92$, and 2.20. (E) EPR spectrum of the rhombic species obtained by subtraction of a spectrum of [CoZn(AAP)] at $12 \mathrm{~K}$ with a $2 \mathrm{~mW}$ microwave power (not shown) from spectrum A. (F) Simulation of spectrum $\mathrm{E}$ with $g_{\text {eff }}$ values of $6.88,2.75$, and $1.80\left(A_{z}=\right.$ $7.0 \mathrm{mT}$ ). For spectrum A, 40 spectra were recorded and averaged.

EPR spectra of [CoZn(AAP)] and [ZnCo(AAP)] were observable under only a very narrow range of EPR operating parameters at low temperatures. These data are consistent with the assignment of the transitions to the ground state doublet since above $30 \mathrm{~K}$ relaxation broadening sets in very rapidly and signals due to [CoZn(AAP)] were lost. The lowest power at which sufficient signal intensity could be obtained in a reasonable time for routine work, $0.2 \mathrm{~mW}$, provided a signal that was slightly saturated (Bennett \& Holz, 1997). In an attempt to further differentiate the broad axial signal observed for [CoZn(AAP)] from the rather similar one of [CoCo(AAP)], the temperature dependence of the signals was investigated (Figure 5). While the intensity of the EPR signal from [CoCo(AAP)] obeys $1 / T$ dependence over only a very narrow temperature range (Bennett \& Holz, 1997), that of [CoZn(AAP)] exhibits ideal behavior at significantly higher temperatures presumably due to the lack of a spin-spin relaxation pathway such as that found with the two interacting paramagnetic Co(II) ions in [CoCo(AAP)]. The possibility that either of the featureless axial signals observed in the [CoZn(AAP)] and [ZnCo(AAP)] spectra could be due to free Co(II) in buffer was also examined. A spectrum of $\mathrm{CoCl}_{2}$ in Hepes buffer at $\mathrm{pH} 7.5$ was recorded under conditions identical to those of [CoZn(AAP)] and [ZnCo(AAP)] and was found to exhibit a broad isotropic signal with markedly different EPR parameters compared to that of [CoZn(AAP)]. Simulation of this spectrum provided $g_{\text {eff }}$ values of 2.25 (2.25), 4.04 (4.04), and 5.08 (5.08). These values correspond to an $M_{s}=| \pm 1 / 2\rangle$ ground state transition with a $g_{\text {real }}$ of 2.29 and an $E / D$ of 0.08 . Even more distinctive was the low-field EPR absorption of $\left[\mathrm{Co}\left(\mathrm{H}_{2} \mathrm{O}\right)_{6}\right]^{2+}$ which extended into zero field, unlike any of the signals observed from cobalt-substituted AAP.

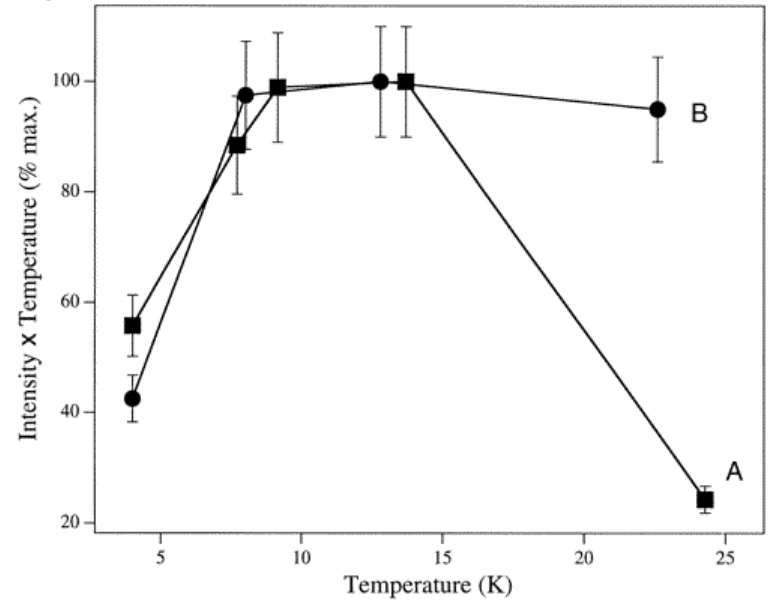

Figure 5 Temperature-weighted EPR intensity dependence upon temperature for (A) [CoCo(AAP)] and (B) [CoZn(AAP)]. Experimental spectra were recorded using a $0.2 \mathrm{~mW}$ microwave power, a $1.26 \mathrm{mT}$ field modulation amplitude, a $100 \mathrm{kHz}$ modulation frequency, and a $10 \mathrm{mT} \mathrm{s}^{-1}$ field sweep rate.

EPR Studies of [CoZn $(A A P)]$ and $[\mathrm{ZnCO}(A A P)]$ at $p H$ 10.0. The fact that two species were observed for both [CoZn(AAP) and [ZnCo(AAP)] raises the following question. What gives rise to these two species? To determine if an ionizable group such as a water/hydroxide equilibrium might be responsible for each species, we recorded EPR spectra of [CoZn(AAP)] (Figure 6A) and [ZnCo(AAP)] (Figure 6C) at pH 10. For [CoZn(AAP)], the species exhibiting ${ }^{59} \mathrm{Co}$ hyperfine structure becomes much more intense and was the dominant species at high $\mathrm{pH}$. The observed EPR spectrum of [CoZn(AAP)] at $\mathrm{pH} 10.0$ was simulated using an $\sim 65 \%$ contribution from a species with $g_{\text {eff }}$ values for $g_{\text {eff }(x, y, z)}$ of $1.80(1.92), 2.75(2.66)$, and $6.88(6.88)$ and an $A_{z}\left({ }^{59} \mathrm{Co}\right)$ of $7.0 \mathrm{mT}(E / D=0.32)$ and an $\sim 35 \%$ contribution from a second species with $g_{\text {eff }}$ values for $g_{\text {eff( }(x, y, z)}$ of 2.20 (2.22), 3.92 (3.87), and 5.23 (5.22) $(E / D=0.1)$ (Figure 6B). These two species are, in fact, identical to those characterized at $\mathrm{pH} 7.5$, and no new species were needed to simulate the EPR spectrum of [CoZn(AAP)] at pH 10.0; however, the relative intensities of these two species were markedly different. That experimental spectra of samples at pH 7.5 and 10 
can both be simulated using these two species only in different relative proportions clearly confirms the assignments of the features in the spectra to individual species. The spectrum of [ZnCo(AAP)] (Figure 6C) recorded at $\mathrm{pH} 10$ was relatively unchanged from that observed at $\mathrm{pH} 7.5$ except for a small increase in the ${ }^{59} \mathrm{Co}$ hyperfine signal. These data suggest that the first metal binding site contains an ionizable group that deprotonates over the $\mathrm{pH}$ range studied while the second site appears to be largely insensitive to $\mathrm{pH}$ changes.

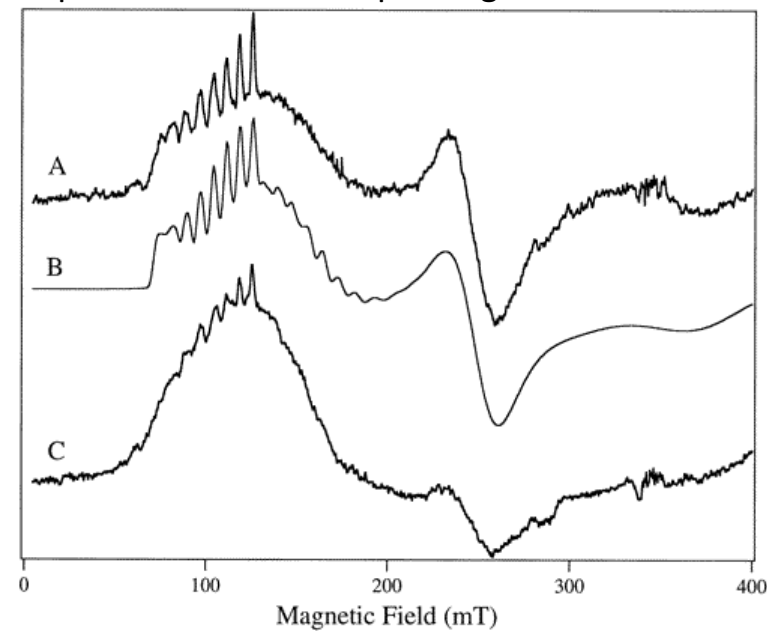

Figure 6 EPR spectra of [CoZn(AAP)] and [ZnCo(AAP)] at pH 10.0 in $50 \mathrm{mM}$ Caps buffer and 20\% 2-propanol. (A) EPR spectrum of [CoZn(AAP)] recorded at $12 \mathrm{~K}$ with a $0.2 \mathrm{~mW}$ microwave power, a $1.26 \mathrm{mT}$ field modulation amplitude, a $100 \mathrm{kHz}$ modulation frequency and a $10 \mathrm{mT} \mathrm{s}^{-1}$ field sweep rate. (B) Simulation of spectrum A obtained by summing the individual simulations of spectra $D(15 \%)$ and $F(85 \%)$ from Figure 4. (C) EPR spectrum of [ZnCo(AAP)] recorded at $12 \mathrm{~K}$ with a $0.2 \mathrm{~mW}$ microwave power, a $1.26 \mathrm{mT}$ field modulation amplitude, a $100 \mathrm{kHz}$ modulation frequency, and a $10 \mathrm{mT} \mathrm{s}^{-1}$ field sweep rate.

Interaction of 1-Butaneboronic Acid with [CoZn $(A A P)]$ and [ZnCo(AAP)]. In order to probe the mechanism of peptide hydrolysis by AAP, we studied the effect of 1-butaneboronic acid (BuBA) on the EPR spectra of [CoZn(AAP)] and [ZnCo(AAP)]. BuBA was previously shown to be a strong competitive inhibitor of native [ZnZn(AAP)] $\left(K_{\mathrm{i}}=10 \mu \mathrm{M}\right.$ at pH 8.0) (Baker \& Prescott, 1983). EPR spectra of [CoZn(AAP)] and [ZnCo(AAP)] were recorded in the presence of 10 equiv of $\mathrm{BuBA}$ at pH 7.5 (Figure 7B,D). For [CoZn(AAP)], a distinct change in the EPR spectrum was evident after as little as $10 \mathrm{~s}$ of incubation time with BuBA. Further incubation of up to $24 \mathrm{~h}$ at 20-25 ${ }^{\circ} \mathrm{C}$ resulted in no further change in the EPR spectrum. In contrast to that of [CoZn(AAP)], the EPR spectrum of [ZnCo(AAP)] at pH 7.5 (Figure 7D) was completely unperturbed in the presence of 10 equiv of BuBA. These results are consistent with the previously reported electronic absorption spectra of [CoZn(AAP)] and [ZnCo(AAP)] in the presence of BuBA. For [CoZn(AAP)], new bands at 480, 500, and $590 \mathrm{~nm}$ were observed with a concomitant increase in the molar absorptivity $\left(\varepsilon_{527}=55 \mathrm{M}^{-1} \mathrm{~cm}^{-1}\right)$, while that of [ZnCo(AAP)] remained unchanged (Prescott et al., 1985). Combination of the EPR spectra reported herein and the previously reported electronic absorption data indicates that BuBA coordinates only to the first metal binding site in AAP and does not interact with the second site. Further, the differential effects of BuBA on the EPR spectra of [CoZn(AAP)] and $[\mathrm{ZnCo}(\mathrm{AAP})]$ again demonstrate that these species are distinct.

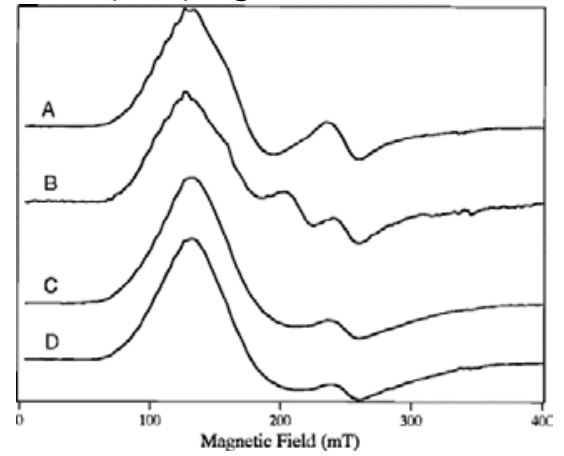

Figure 7 EPR spectra of (A) [CoZn(AAP)] and (C) [ZnCo(AAP)] at pH 7.5 in $50 \mathrm{mM}$ Hepes buffer and 20\% 2-propanol. (B) EPR spectrum of a $1 \mathrm{mM}$ [CoZn(AAP)] sample in $50 \mathrm{mM}$ Hepes buffer and 20\% 2-propanol at pH 7.5 after the addition of $10 \mathrm{mM}$ BuBA 
at $25^{\circ} \mathrm{C}$ for $24 \mathrm{~h}$. (D) EPR spectrum of a $1 \mathrm{mM}$ [ZnCo(AAP)] sample in $50 \mathrm{mM}$ Hepes buffer and 20\% 2-propanol at pH 7.5 after incubation with $10 \mathrm{mM}$ BuBA at $25^{\circ} \mathrm{C}$ for $24 \mathrm{~h}$. All spectra were recorded at $12 \mathrm{~K}$ with a $0.2 \mathrm{~mW}$ microwave power, a $1.26 \mathrm{mT}$ field modulation amplitude, a $100 \mathrm{kHz}$ modulation frequency, and a $10 \mathrm{mT} \mathrm{s}^{-1}$ field sweep rate.

From difference spectroscopy and from simulation of the [CoZn(AAP)] EPR spectrum at pH 7.5 (Figure 4), it appeared that the observed change was due to a third species. This third species was isolated by subtraction of the spectrum [CoZn(AAP)] from that of [CoZn(AAP)] + BuBA (Figure 8A). This signal was simulated with $g_{\text {eff }}$ values for $g_{\mathrm{eff}(x, y, z)}$ of 2.08 (2.08), 3.15 (3.15), and 6.15 (6.15) which correspond to an $M_{\mathrm{s}}=| \pm 1 / 2\rangle$ ground state transition with a $g_{\text {real }}$ of 2.41 and an $E / D$ of 0.22 . This simulation also invoked an eight-line unresolved ${ }^{59} \mathrm{Co}$ hyperfine pattern with an $A_{2}\left({ }^{59} \mathrm{Co}\right)$ value of $4.0 \mathrm{mT}$ (Figure $\left.8 \mathrm{~B}\right)$. Summation of these three species provides the simulation of the observed [CoZn(AAP)] + BuBA EPR spectrum (Figure 9B). Simulations not including a third signal with a hyperfine component of this order were wholly unsatisfactory. While this signal superficially appears somewhat unusual compared to the other observed signals from mono-Co(II) ions in substituted forms of AAP, the relatively high $g_{\text {real }}$ and large $E / D$ values are actually a characteristic of the observed ${ }^{59}$ Co hyperfine split signals from Co(II)-bound AAP.

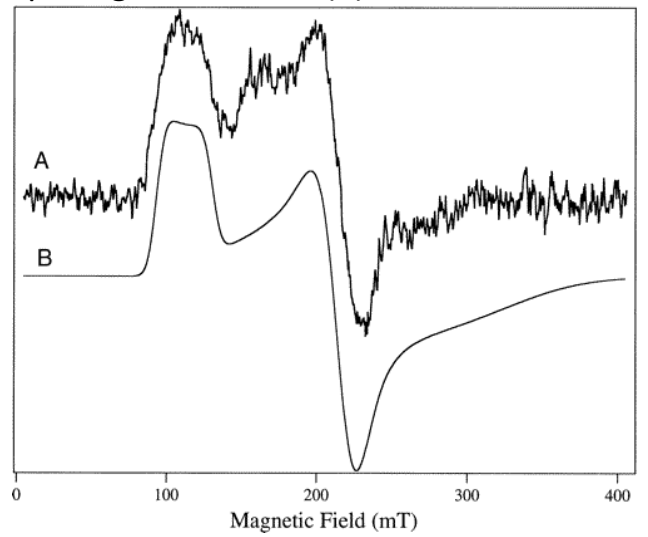

Figure 8 (A) EPR spectrum of a rhombic species obtained by subtraction of the EPR spectrum of $1 \mathrm{mM}$ samples of [CoZn(AAP)] in $50 \mathrm{mM}$ Hepes buffer and 20\% 2-propanol at pH 7.5 in the absence and presence of $10 \mathrm{mM} \mathrm{BuBA}$. (B) Simulation of spectrum A with $g_{\text {eff }}$ values of $6.15,3.15$, and $2.08\left(A_{z}=4.0 \mathrm{mT}, g_{\text {real }}=2.41\right.$, and $\left.E / D=0.22\right)$. The EPR spectrum of [CoZn(AAP)] was recorded at $12 \mathrm{~K}$ with a $0.2 \mathrm{~mW}$ microwave power, a $1.26 \mathrm{mT}$ field modulation amplitude, a $100 \mathrm{kHz}$ modulation frequency, and a $10 \mathrm{mT} \mathrm{s}$ 1 field sweep rate.

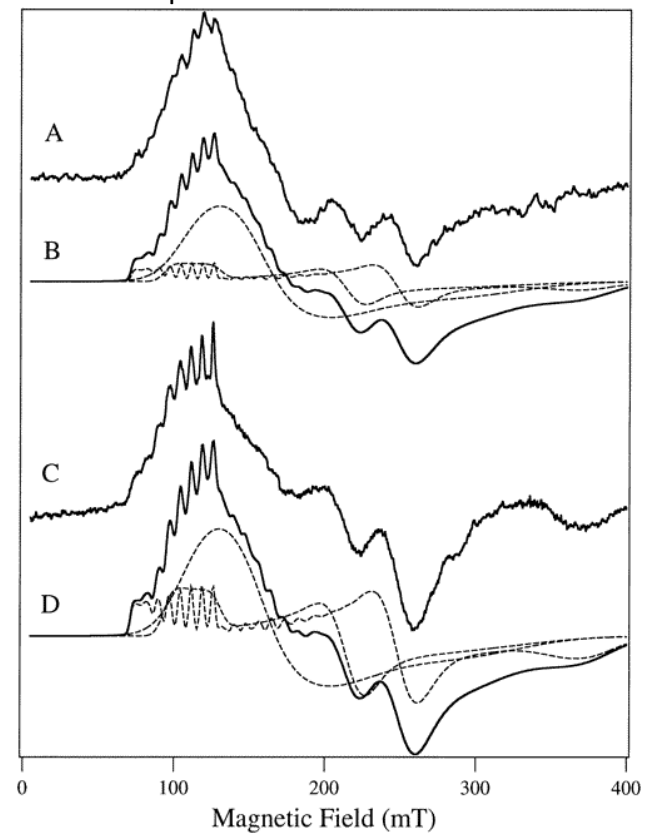

Figure 9 (A) EPR spectrum of [CoZn(AAP)] in 50 mM Hepes buffer and 20\% 2-propanol at pH 7.5 after the addition of $10 \mathrm{mM}$ BuBA at $25^{\circ} \mathrm{C}$ for $24 \mathrm{~h}$. (B) Simulation of spectrum A obtained by summing the individual simulations of [CoZn(AAP)] (Figure 4D,F) with 
the simulation shown in Figure 8 in a 85\%: 15\% ratio. (C) EPR spectrum of [CoZn(AAP)] in 50 mM Caps buffer and 20\% 2-propanol at $\mathrm{pH} 7.5$ after the addition of $10 \mathrm{mM} \mathrm{BuBA}$ at $25^{\circ} \mathrm{C}$ for $24 \mathrm{~h}$. (D) Simulation of spectrum C obtained by summing the individual simulations of [CoZn(AAP)] (Figure 4D, F) with the simulation shown in Figure 8 in a 65\%: $35 \%$ ratio. All spectra were recorded at $12 \mathrm{~K}$ with a $0.2 \mathrm{~mW}$ microwave power, a $1.26 \mathrm{mT}$ field modulation amplitude, a $100 \mathrm{kHz}$ modulation frequency, and a $10 \mathrm{mT} \mathrm{s}$ ${ }^{1}$ field sweep rate.

EPR spectra of [CoZn(AAP)] in the presence of 10 equiv of BuBA were recorded at $\mathrm{pH} 10.0$ (Figure 9C). The two species exhibiting ${ }^{59} \mathrm{Co}$ hyperfine structure become markedly more intense at high $\mathrm{pH}$. The observed EPR spectrum was simulated using an $\sim 65 \%$ contribution from the two species with ${ }^{99} \mathrm{Co}$ hyperfine structure and an $\sim 35 \%$ contribution from the third more axial species (Figure 9D). These three species exhibited $g_{\text {eff }}$ values identical to those used to simulate the [CoZn(AAP)] + BuBA spectrum recorded at pH 7.5. Therefore, no new species were needed to simulate the EPR spectrum of [CoZn(AAP)] + BuBA at pH 10.0. Attempts to increase the proportion of the third, BuBA-dependent $g_{\text {real }}=2.41$ EPR species by adding more BuBA were thwarted by the poor solubility of BuBA. The EPR spectrum of [ZnCo(AAP)] upon addition of BuBA at pH 10.0 was, like the signal at $\mathrm{pH} 7.5$, completely unperturbed.

\section{Discussion}

Several aminopeptidases, including AAP, are members of a growing class of metallohydrolases that contain two metal ions in the enzyme active site (Vallee \& Auld, 1993a,b; Dismukes, 1996; Lipscomb \& Sträter, 1996; Sträter et al., 1996; Wilcox, 1996). For AAP, the two Zn(II) ions are essential for full enzymatic activity; however, the addition of $1 \mathrm{~mol}$ of $\mathrm{Zn}(\mathrm{II})$ to apo-AAP provides an enzyme with $c a$. $80 \%$ of its native enzymatic activity. Substitution of one or both $\mathrm{Zn}(\mathrm{II})$ ions in AAP with other divalent first row transition metal ions provides activities of different magnitudes that are dependent on the sequence and order of addition (Prescott et al., 1983, 1985; Bayliss \& Prescott, 1986). Therefore, both metal ions are required for full enzymatic activity, but the exact roles of each metal ion in catalysis are unknown. As isolated, AAP possesses a $(\mu$-aqua) $(\mu-$ carboxylato)dizinc(II) core with one terminal carboxylate and one histidine residue at each metal site (Chevrier et al., 1994). In order to gain insight into the role of each metal ion in catalysis, EPR spectra of the [CoZn(AAP)] and $[\mathrm{ZnCo}(\mathrm{AAP})]$ metal-substituted enzymes were recorded at $\mathrm{pH}$ 7.5. The spectra obtained clearly show that the metal-binding sites in AAP can be selectively labeled and, depending on which site the Co(II) ion occupies, distinct EPR signatures are observed.

Inspection of the observed EPR spectra for [CoZn(AAP)] and [ZnCo(AAP)] indicates that two species are present in solution and that these two species can be differentiated for [CoZn(AAP)] and simulated. Solely on the basis of the EPR data, the coordination number and geometry of the Co(II) ions in [CoZn(AAP)] and [ZnCo(AAP)] cannot be determined. As Johnson and co-workers pointed out, the presence or absence of resolvable ${ }^{59} \mathrm{Co}$ hyperfine structure is not an indication of coordination geometry since both tetrahedral and octahedral complexes exhibit ${ }^{59}$ Co hyperfine structure (Werth et al., 1995). Moreover, Pilbrow and Hanson (1993) showed theoretically that the magnitude of ${ }^{9} \mathrm{Co}$ hyperfine structure is effectively independent of the Co(II) ion coordination geometry. Empirical relationships between EPR parameters and coordination geometry for highspin $S=3 / 2 \mathrm{Co}$ (II) ions are also difficult to deduce, primarily because of a lack of reliable EPR parameters for $\mathrm{Co}$ (II) coordination complexes in the literature. Therefore, the only direct geometrical information available from EPR is the extent of deviation from idealized axial geometry as indicated by the rhombic distortion, $E / D$, of the axial zero field splitting (Figure 1). Since a new systematic protocol for the simulation of high-spin $S=3 /{ }_{2} \operatorname{Co}(I I)$ EPR spectra was recently reported (Bennett \& Holz, 1997), E/D values can now be easily obtained from the relationships expressed in eqs 1-3 and illustrated in Figure 2.

Comparison of the EPR data of [CoZn(AAP)] and [ZnCo(AAP)] with the previously reported electronic absorption data suggests that the difference between the two EPR active species in each sample is not likely one 
of coordination number. This must be the case for [ZnCo(AAP)] since the Co(II) ion clearly resides in an octahedral environment with a small $\left(\sim 10 \mathrm{M}^{-1} \mathrm{~cm}^{-1}\right)$ molar absorptivity. The two species observed in the EPR spectra of [CoZn(AAP)] and [ZnCo(AAP)] appear to be the result of constraints imposed on the Co(II) ion coordination geometry by the ligand field. For example, the signal exhibiting resolvable ${ }^{9} \mathrm{Co}$ hyperfine splitting is likely due to a Co(II) ion in a highly constrained and asymmetric coordination geometry. That the geometry is asymmetric is clearly evident from the anisotropy of the EPR spectrum $(E / D=0.32)$. Furthermore, the high $g_{\text {real }}$ value of 2.54 is indicative of a high degree of spin-orbit coupling, as would be expected for a high-spin $\mathrm{Co}(\mathrm{II})$ ion in a coordination environment with low symmetry. A highly constrained geometry is also evident from the resolvable ${ }^{59} \mathrm{Co}$ hyperfine splitting in that the EPR line width has to be narrow enough and not significantly broadened by factors such as $g$-strain which arise from microdistributions in the Co(II) coordination environment. In the case of the ${ }^{9} \mathrm{Co}$ hyperfine-split signal, it is evident that the variability in the structure of the species responsible must be small, again indicating a highly constrained Co(II) environment.

In contrast, the second species appears to be due to a Co(II) center with much less constraint upon its geometry. The less rhombically distorted EPR spectrum $(E / D=0.1)$ is an indication of a significantly more symmetrical environment. Such an environment is likely the result of relaxation of ligand sphere-derived constraints. Consistent with the adoption of such a coordination geometry being due to the relaxation of the ligand field, a wider distribution of microheterogeneous structures in a given sample is observed. The deviation of $g_{\text {real }}=2.25$ from the free electron value of 2.0 is required by spin-orbit coupling, which will be uniformly small in axially symmetric systems. In most cases, axial symmetry will be adopted by systems with a high degree of flexibility that allows the coordination geometry to adopt the lowest energy. However, a number of other slightly higher energy conformations exist into which the complex can be excited by thermally induced vibrational modes. It is reasonable to expect that, for an axially symmetric Co(II) species, the distribution of microheterogeneous environments will be relatively wide and the resultant $g$ strain in the EPR spectrum will preclude the observation of ${ }^{59} \mathrm{Co}$ hyperfine splitting, although the hyperfine interaction is still present. It is worth noting that although the broad axial species of [CoZn(AAP)] superficially may appear to resemble signals observed in other $\mathrm{Co}(\mathrm{II})$ systems, including $[\mathrm{CoCo}(\mathrm{AAP})]$ and $\left[\mathrm{Co}\left(\mathrm{H}_{2} \mathrm{O}\right)_{6}\right]^{2+}$, computer simulation has shown that each of these signals has distinct EPR parameters. The $\mathrm{pH}$ dependencies of the signals from the three $\mathrm{Co}$ (II)substituted AAP derivatives are all different, as are their temperature dependencies, and the signal from $\left[\mathrm{Co}\left(\mathrm{H}_{2} \mathrm{O}\right)_{6}\right]^{2+}$ is distinguished by a very low-field absorption not observed in any of the AAP samples. The similarity in the appearance of these signals should not be overinterpreted since the EPR spectra of any essentially axial $\mathrm{Co}(\mathrm{II})$ ion will closely resemble each other because the $g_{\text {eff }}$ values are dictated by $g_{\text {real }}$ and $E / D$. This is illustrated by noting the similarity between the spectra of [CoCo(AAP)] (Bennett \& Holz, 1997), [CoZn(AAP)] at pH 7.5, $\left[\mathrm{Co}\left(\mathrm{H}_{2} \mathrm{O}\right)_{6}\right]^{2+}, \mathrm{Co}(\mathrm{II})$-substituted R2 (Elgren et al., 1994), and the $\mathrm{Co}(\mathrm{II})$-substituted $\beta$-lactamase from Bacteroides fragilis (Crowder et al., 1996). These species have similar EPR signals despite differences in coordination geometry, coordination number, and types of ligand atoms. Therefore, correlation of either coordination number or geometry in species exhibiting similar EPR spectra should not be assumed, whereas the symmetry of the electronic field can be inferred.

Further information from EPR concerning the environment of the Co(II) ions in either the first or second metal binding sites of AAP is available by inspecting the spectra recorded at different $\mathrm{pH}$ values. EPR spectra of [CoZn(AAP)] recorded at high $\mathrm{pH}$ show that the relative proportion of the two species in solution is $\mathrm{pH}$ dependent and suggest that at least one ionizable group interacts with the Co(II) ion in [CoZn(AAP)] (Scheme 1). On the other hand, the EPR spectrum of [ZnCo(AAP)] is much less dramatically affected upon raising the $\mathrm{pH}$ and the essentially axial, featureless species remains dominant. Comparison of the EPR signals observed for [CoZn(AAP)] and [ZnCo(AAP)] at pH 10 with those observed for [Co_(AAP)] and [CoCo(AAP)] at pH 10 (Bennett \& Holz, 1997) reveals some striking similarities and differences. Similar to that of [CoZn(AAP)], EPR spectra of [Co_(AAP)] recorded at pH 10 exhibited a marked increase in the ${ }^{59} \mathrm{Co}$ hyperfine signal and a decrease in the intensity of the broad axial signal. On the other hand, EPR spectra of [CoCo(AAP)] recorded at pH 10 were 
indistinguishable from those recorded at $\mathrm{pH}$ 7.5. In addition, as the $\mathrm{pH}$ was increased from 5.9 to 10.0 , the intensity of an $S=3$ parallel mode EPR signal, due to ferromagnetic coupling between the two Co(II) ions, decreased (Bennett \& Holz, 1997). Concomitant with the loss of the $S=3$ signal, an increase in the $S=3 / 2$ signal due to [CoCo(AAP)] was observed. Therefore, an ionizable group perturbs the metal ion bound in the first metal binding site but has little effect on the second metal binding site. This ionizable group also mediates spin coupling between the two Co(II) centers in the absence of substrate since the $S=3$ species giving rise to the parallel mode signal is lost upon raising the $\mathrm{pH}$ or by adding BuBA.

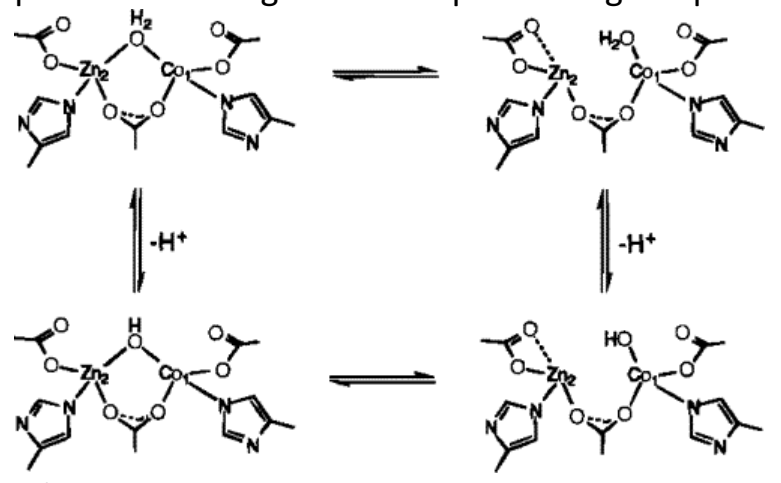

Scheme 1

Inspection of the X-ray crystal structure of AAP reveals that a single oxygen atom bridges the two $\mathrm{Zn}$ (II) ions in the native enzyme at pH 7.0 (Chevrier et al., 1994, 1996), and kinetic studies indicate that this bridging water/hydroxide has a $\mathrm{p} K_{\mathrm{a}}$ of $\sim 7$ (Chen et al., 1997). One possible explanation for the presence of two species in $\mathrm{Co}(\mathrm{II})$-substituted AAP would be the existence of a bridging water molecule that is in equilibrium with a bridging or terminal hydroxide (Scheme 1). If so, as the $\mathrm{pH}$ is increased, a higher proportion of the Co(II) ions in a population of [CoZn(AAP)] interacts with a hydroxyl group and this interaction constrains the geometry of the $\mathrm{Co}(\mathrm{II})$ ion. This proposal is consistent with the EPR data in that a greater percentage of the ${ }^{59} \mathrm{Co}$ hyperfine-split signal is observed at high $\mathrm{pH}$ values for both [Co_(AAP)] and [CoZn(AAP)]. A hydroxyl group will have a smaller dissociation constant than water and would, therefore, be capable of constraining the Co(II) geometry far more effectively than water. Thus, at low $\mathrm{pH}$ values, the existence of a water ligand predominates and the Co(II) ion is afforded a flexibility that is lost upon raising the $\mathrm{pH}$ to a point where the much stronger binding hydroxyl predominates. This effect is significantly less observable with $\mathrm{Co}(\mathrm{II})$ in the second metal binding site. The stronger interaction of a hydroxyl ion with the metal ion in the first binding site may be related to its specificity for that metal since AAP is $\sim 80 \%$ active with only one metal ion present. Therefore, the $\mathrm{pH}$ dependence observed in the EPR spectra of [CoZn(AAP)] and [ZnCo(AAP)] appears to be due to the deprotonation of a bridging water moiety to a bridging or terminal hydroxide.

The following question is important for understanding the mechanism of action of AAP and dinuclear hydrolases in general. How does substrate interact with the dimetal cluster? BuBA was previously reported to be a strong competitive inhibitor of AAP $\left(K_{\mathrm{i}}=10 \mu \mathrm{M}\right.$ at pH 8.0) (Baker \& Prescott, 1983). Prescott et al. (1985) suggested from electronic absorption data that BuBA bound only to the first metal binding site. The present EPR studies are entirely in agreement with this proposal since addition of BuBA to [CoZn(AAP)] clearly perturbs the EPR spectrum. The complete lack of any effect of BuBA on the EPR spectrum or the electronic absorption spectrum of [ZnCo(AAP)] (Prescott et al., 1985) suggests that BuBA does not interact with the second metal ion. The fact that BuBA addition to [CoZn(AAP)] does not result in a single species suggests that the $K_{\mathrm{i}}$ for BuBA binding is greater than that observed for [ZnZn(AAP)]. The consequence of a substantially larger $K_{i}$ for BuBA binding to [CoZn(AAP)] would be that an equilibrium mixture of BuBA-bound and unbound enzyme would exist in solution. Simulation of the EPR spectrum of [CoZn(AAP)] in the presence of BuBA indicates that this is precisely the situation. Inspection of the recently reported X-ray crystal structure of the bovine lens leucine aminopeptidase (bILAP) with the transition state analog inhibitor L-leucinephosphonic acid (LPA) bound indicates that LPA provides a single oxygen atom bridge between the two Zn(II) ions (Sträter \& Lipscomb, 1995). Similarly, 
the X-ray crystal structure of the transition state analog inhibitor D-iodophenylalanine hydroxamate bound to AAP also indicates that a single oxygen atom bridge is provided between the two $\mathrm{Zn}$ (II) ions (Chevrier et al., 1996). Therefore, in the transition state, a single oxygen atom likely bridges the two metal ions which is clearly not the case upon BuBA binding. Since BuBA binds only to the first metal binding site, it appears to behave as a substrate-like inhibitor rather than like a transition state analog inhibitor as previously suggested (Baker \& Prescott, 1983).

The observation that the two species exhibiting ${ }^{59} \mathrm{Co}$ hyperfine structure in [CoZn(AAP)] become markedly more intense at high $\mathrm{pH}$ suggests that BuBA binds only to the Co(II) ions that contain a bridging or terminal hydroxide moiety. On the basis of X-ray crystallographic data of a transition state analog inhibitorbound AAP complex, the carbonyl oxygen of the peptide indeed binds directly to only one metal ion (Scheme 2) (Chevrier et al., 1996). This binding scheme is consistent with the large negative entropy and large positive enthalpy of activation reported for AAP (Chen et al., 1997). Since AAP is ca. $80 \%$ active with only a single $\mathrm{Zn}$ (II) ion bound and recent fluoride inhibition studies indicate that fluoride binding occurs only after substrate binding (Chen et al., 1997), the bridging water/hydroxide moiety likely becomes terminal at the same time as substrate binding and appears to represent the hydroxylating agent in the enzymatic reaction (Scheme 2). The EPR data reported herein thus substantiate the fact that substrate binding to the first metal ion is in fact the first step in catalysis, as proposed in the recently reported mechanism of action for AAP (Bennett \& Holz, 1997; Chen et al., 1997).
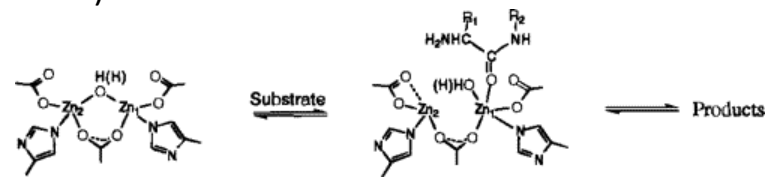

Scheme 2

\section{Acknowledgment}

The Bruker ESP-300E EPR spectrometer was purchased with funds provided by the National Science Foundation (Grant BIR-9413530) and Utah State University. The program Rhombogram was kindly supplied by Professor Wilfred R. Hagen. The program EPRSim XOP was made available by Professor John Boswell (Oregon Graduate Institute).

\section{$X$}

Abstract published in Advance ACS Abstracts, August 1, 1997.

\section{References}

Baker, J. O., \& Prescott, J. M. (1983) Biochemistry 22, 5322-5331.

Bayliss, M. E., \& Prescott, J. M. (1986) Biochemistry 25, 8113-8117.

Beese, L. S., \& Steitz, T. A. (1991) EMBO J. 10, 25-33.

Bennett, B., \& Holz, R. C. (1997) J. Am. Chem. Soc. 119, 1923-1933.

Bennett, B., Benson, N., McEwan, A. G., \& Bray, R. C. (1994a) Eur. J. Biochem. 225, 321-331.

Bennett, B., Berks, B. C., Ferguson, S. J., Thomson, A. J., \& Richardson, D. J. (1994b) Eur. J. Biochem.226, 789-798.

Bennett, B., Gruer, M., Guest, J. R., \& Thomson, A. J. (1995) Eur. J. Biochem. 233, 317-326.

Bertini, I., \& Luchinat, C. (1984) Adv. Inorg. Biochem. 6, 71-111.

Bone, R., Frank, L., Springer, J. P., Pollack, S. J., Osborne, S.-A., Atack, J. R., Knowles, M. R., McAllister, G., Ragan, C. I., Broughton, H. B., Baker, R., \& Fletcher, S. R. (1994) Biochemistry 33, 9460-9467.

Bray, R. C. (1988) Q. Rev. Biophys. 21, 299-329.

Burley, S. K., David, P. R., Taylor, A., \& Lipscomb, W. N. (1990) Proc. Natl. Acad. Sci. U.S.A. 87,6878-6882.

Burley, S. K., David, P. R., Sweet, R. M., Taylor, A., \& Lipscomb, W. N. (1992) J. Mol. Biol. 224, $113-140$.

Chen, G., Edwards, T., D'souza, V. M., \& Holz, R. C. (1997) Biochemistry 36, 4278-4286. 
Chevrier, B., Schalk, C., D'Orchymont, H., Rondeau, J.-M., Moras, D., \& Tarnus, C. (1994) Structure 2,283-291.

Chevrier, B., D'Orchymont, H., Schalk, C., Tarnus, C., \& Moras, D. (1996) Eur. J. Biochem. 237, 393-398.

Chin, J. (1991) Acc. Chem. Res. 24, 145-152.

Crowder, M. W., Wang, Z., Franklin, S. L., Zovinka, E. P., \& Benkovic, S. J. (1996) Biochemistry 35,12126-12132.

Dismukes, G. C. (1996) Chem. Rev. 96, 2909-2926.

Elgren, T. E., Ming, L.-J., \& Que, L. (1994) Inorg. Chem. 33, 891-894.

Griffith, J. P., Kim, J. L., Kim, E. E., Sintchak, M. D., Thomson, J. A., Fitzgibbon, M. J., Fleming, M. A., Caron, P. R., Hsiao, K., \& Navia, M. A. (1995) Cell 82, 507-522.

Holz, R. C., Que, L., Jr., \& Ming, L.-J. (1992) J. Am. Chem. Soc. 114, 4434-4436.

Jabri, E., Carr, M. B., Hausinger, R. P., \& Karplus, P. A. (1995) Science 268, 998-1004.

Kim, E. E., \& Wyckoff, H. W. (1991) J. Mol. Biol. 218, 449-464.

Kim, H., \& Lipscomb, W. N. (1993) Proc. Natl. Acad. Sci. U.S.A. 90, 5006-5010.

Kim, Y., Eom, S. H., Wang, J., Lee, D.-S., Suh, S. W., \& Steitz, T. A. (1995) Nature 376, 612.

Lahm, A., Volbeda, A., \& Suck, D. (1990) J. Mol. Biol. 215, 207-210.

Lai, K., Dave, K. I., \& Wild, J. R. (1994) J. Biol. Chem. 269, 16579-16584.

Lauffer, R. B., Antanaitis, B. C., Aisen, P., \& Que, L., Jr. (1983) J. Biol. Chem. 258, 14212-14218.

Lipscomb, W. N., \& Sträter, N. (1996) Chem. Rev. 96, 2375-2433.

Maurice, A. M. (1980) Ph.D. Thesis, University of Illinois, Urbana, IL.

Menger, F. M., Gan, L. H., Johnson, E., \& Durst, D. H. (1987) J. Am. Chem. Soc. 109, 2800-2803.

Nilges, M. J. (1979) Ph.D. Thesis, University of Illinois, Urbana, IL.

Pilbrow, J. R. (1978) J. Magn. Reson. 31, 479-489.

Pilbrow, J. R. (1990) Transition Ion Electron Paramagnetic Resonance, Oxford University Press, Oxford.

Prescott, J. M., \& Wilkes, S. H. (1976) Methods Enzymol. 45B, 530-543.

Prescott, J. M., Wagner, F. W., Holmquist, B., \& Vallee, B. L. (1983) Biochem. Biophys. Res. Commun. 114,646-652.

Prescott, J. M., Wagner, F. W., Holmquist, B., \& Vallee, B. L. (1985) Biochemistry 24, 5350-5356.

Rattinger, G. B., Belford, R. L., Walker, H., \& Brown, T. L. (1989) Inorg. Chem. 28, 1059-1066.

Roderick, S. L., \& Matthews, B. W. (1993) Biochemistry 32, 3907-3912.

Rose, S. L., Dickinson, L. C., \& Westhead, E. W. (1984) J. Biol. Chem. 259, 4405-4413.

Sears, H. J., Bennett, B., Spiro, S., Thomson, A. J., \& Richardson, D. J. (1995) Biochem. J. 310, 311-314.

Sträter, N., \& Lipscomb, W. N. (1995) Biochemistry 34, 9200-9210.

Sträter, N., Klabunde, T., Tucker, P., Witzel, H., \& Krebs, B. (1995) Science 268, 1489-1492.

Sträter, N., Lipscomb, W. N., Klabunde, T., \& Krebs, B. (1996) Angew. Chem., Int. Ed. Engl. 35, 2024-2055.

True, A. E., Scarrow, R. C., Randall, C. R., Holz, R. C., \& Que, L. J. (1993) J. Am. Chem. Soc. 115,4246-4255.

Vallee, B. L., \& Auld, D. S. (1993a) Proc. Natl. Acad. Sci. U.S.A. 90, 2715-2718.

Vallee, B. L., \& Auld, D. S. (1993b) Biochemistry 32, 6493-6500.

Van Wart, H. E., \& Lin, S. H. (1981) Biochemistry 20, 5682-5689.

Werth, M. T., Tang, S.-F., Formicka, G., Zeppezauer, M., \& Johnson, M. K. (1995) Inorg. Chem. 34,218-228.

Wilcox, D. E. (1996) Chem. Rev. 96, 2435-2458.

(1) Abbreviations: Caps, 3-(cyclohexylamino)-1-propanesulfonic acid; Hepes, 4-(2-hydroxyethyl)-1piperazineethanesulfonic acid; Mops, 3-( $N$-morpholino)propanesulfonic acid; Tricine, $N$ -

tris(hydroxymethyl)methylglycine; BuBA, 1-butaneboronic acid; AAP, aminopeptidase from A. proteolytica; bILAP, bovine lens leucine aminopeptidase; EPR, electron paramagnetic resonance. 